passes, and increased rates of first pass success without increased risk of intracranial hemorrhage. These results are in contrast to the increased hemorrhage rates reported in $\mathrm{AF}$ associated stroke treated with supportive care and or intravenous thrombolysis. Bridging therapy in AF patients undergoing thrombectomy independently increased the odds of intracranial hemorrhage and did not improve functional outcomes. Together, these results suggest that AF associated stroke has a differential response to intravenous thrombolysis and mechanical thrombectomy. Randomized trials are warranted to determine whether patients with $\mathrm{AF}$ associated stroke may benefit by deferring bridging therapy at thrombectomy-capable centers.

Disclosures F. Akbik: None. A. Alawieh: None. C. Cawley: None. B. Howard: None. F. Tong: None. F. Nahab: None. F. Nahab: None. H. Saad: None. L. Dimisko: None. O. Samuels: None. G. Pradilla: None. I. Maier: None. W. Feng: None. R. Chalhoub: None. N. Goyal: None. R. Starke: None. A. Rai: None. K. Fargen: None. M. Psychogios: None. P. Jabbour: None. R. De Leacy: None. S. Keyrouz: None. T. Dumont: None. P. Kan: None. J. Liman: None. A. Arthur: None. D. Mccarthy: None. V. Saini: None. S. Wolfe: None. J. Mocco: None.

\section{LB-003 ISCHEMIC STROKE ASSOCIATED WITH COVID-19 AND RACIAL OUTCOME DISPARITY IN NORTH AMERICA}

${ }^{1} \mathrm{~A}$ Dmytriw ${ }^{*},{ }^{1} \mathrm{~K}$ Phan, ${ }^{2} \mathrm{C}$ Schirmer, ${ }^{3} \mathrm{~F}$ Settecase, ${ }^{3} \mathrm{M}$ Heran, ${ }^{4} \mathrm{~A}$ Efendizade, ${ }^{5} \mathrm{~A}$ Kuhn, ${ }^{5} \mathrm{~A}$ Puri, ${ }^{6} \mathrm{~B}$ Menon, ${ }^{7} \mathrm{M}$ Dibas, ${ }^{8} \mathrm{~S}$ Sivakumar, ${ }^{9} \mathrm{~A}$ Mowla, ${ }^{10} \mathrm{~L}$ Leung, ${ }^{10} \mathrm{~A}$ Malek, ${ }^{11} \mathrm{~B}$ Voetsch, ${ }^{11} \mathrm{~S}$ Segal, ${ }^{11} \mathrm{~A}$ Wakhloo, ${ }^{12} \mathrm{H}$ Wu, ${ }^{13} \mathrm{~A}$ Xavier, ${ }^{12} \mathrm{~A}$ Tiwari. ${ }^{1}$ Harvard University, Boston, MA, Canada; ${ }^{2}$ Geisinger, Wilkes-Barre, PA; ${ }^{3}$ Vancouver General Hospital, Vancouver, $B C$, Canada; ${ }^{4}$ SUNY Downstate Hospital, New York, NY; ${ }^{5}$ UMass Memorial Medical Center, Worcester, MA; ${ }^{6}$ Calgary Cumming School of Medicine, Calgary, AB, Canada; ${ }^{7}$ Harvard University, Boston, MA; ${ }^{8}$ Prisma Health, Greenville, SC; ${ }^{9}$ Keck School of Medicine USC, Los Angeles, CA; ${ }^{10}$ Tufts University Hospital, Boston, MA; ${ }^{11}$ Lahey Hospital Health Center, Burlington, MA; ${ }^{12}$ Brookdale University Hospital, Brooklyn, NY; ${ }^{13}$ Sinai Grace Hospital, Detroit, MI

\subsection{6/neurintsurg-2020-SNIS.275}

Introduction There has been limited evidence on the influence of racial background in stroke outcomes in COVID-19. Underlying biological, genetic, or epigenetic characteristics may predispose to health differences and outcomes. Social determinants of health, access and geographical differences pertaining both to population density and other location-based factors may also be important.

Methods We report 69 cases of acute stroke in patients positive for SARS-CoV-2, including 27 of African American background and 42 of other racial backgrounds, including Caucasian, Hispanic, and Asian. All patients presented to 14 major hospitals in the United States and Canada, from March 14-April 14, 2020. All patients had nasopharyngeal swab samples that were positive for SARS-CoV-2 on qualitative RT-PCR assays. We present a dichotomized analysis of ischemic stroke outcomes between patients of African American background as reported on hospital intake questionnaire versus all other backgrounds.

Results Comparison between Caucasian, Hispanic and Asian backgrounds did not show disparities in stroke outcomes. We found no significant difference in age (64.4 versus 62.9 years) or the proportion of females $(51.9 \%$ versus $38.1 \%)$ (table 1). Diabetes mellitus was present significantly less in African American cases versus others (37\% vs. 66.7\%). No significant difference between groups was found regarding other comorbidities including smoking, atrial fibrillation, prior anticoagulation, coronary artery disease, congestive heart failure, hypertension, hyperlipidemia, cerebrovascular accident, peripheral vascular disease, or chronic kidney disease. With respect to presenting SARS-CoV-2 symptoms, we found no difference in exposure history, asymptomatic cases, fever, cough, dyspnea, nausea or vomiting, chills, malaise, or lethargy. The African American cohort had a similar mean NIHSS score of 16.3 compared with 14.9 in other races $(p=0.63)$. The door-to-CT time was also similar (23 versus 19 minutes). The proportion of patients presenting with a large vessel occlusion was not significantly different $(40.7 \%$ versus $47 \%$ ). We noted $14.8 \%$ of African American cases received intravenous tPA compared to $31 \%$ in other races, but this was not significantly different in this sample. The proportion of thrombectomy cases mirrored this $(14.8 \%$ versus $31 \%$ ). Laboratory findings were not significantly different between African Americans and all others. Regarding stroke functional outcomes, there was no difference between African Americans and other races in terms of discharge mRS $(\mathrm{p}=0.27)$. For mRS $0-2$, there was no significant difference noted $(14.8 \%$ versus $16.7 \%)$. Symptomatic intracranial hemorrhage (sICH) was significantly higher for African Americans $(11.1 \%$ versus $3 \%, p<0.001)$. Mortality was significantly higher in African Americans compared to other races $151.9 \%$ vs. $28.6 \%, \mathrm{p}=0.03)$.

Discussion Our preliminary data suggest that there may be a mortality difference amongst stroke patients of African American background afflicted with COVID-19. The reasons for increased mortality in African Americans with COVID-19-associated stroke are unknown. Racial disparities in case counts and outcomes during the COVID-19 pandemic have been highlighted, particularly regarding African American communities. In addition, the finding in this study that mortality rate of COVID-19 positive stroke patients is greater than that previously reported in either COVID-19 respiratory infection alone or acute ischemic stroke alone, suggests an interaction that also warrants further study.

Disclosures A. Dmytriw: None. K. Phan: None. C. Schirmer: None. F. Settecase: None. M. Heran: None. A. Efendizade: None. A. Kuhn: None. A. Puri: None. B. Menon: None. M. Dibas: None. S. Sivakumar: None. A. Mowla: None. L. Leung: None. A. Malek: None. B. Voetsch: None. S. Segal: None. A. Wakhloo: None. H. Wu: None. A. Xavier: None. A. Tiwari: None.

\section{LB-004 SEX DIFFERENCES IN ACUTE STROKE THROMBECTOMY MANAGEMENT AND OUTCOMES IN THE UNITED STATES: EVIDENCE FROM THE NVQI-QOD REGISTRY}

${ }^{1} \mathrm{~V}$ Thirunavu ${ }^{*},{ }^{2} \mathrm{R}$ Abdalla, ${ }^{2} \mathrm{D}$ Cantrell, ${ }^{3} \mathrm{M}$ Hurley, ${ }^{3} \mathrm{~A}$ Shaibani, ${ }^{1} \mathrm{~B}$ Jahromi, ${ }^{1} \mathrm{M}$ Potts, ${ }^{4} S$ Ansari, ${ }^{5} \mathrm{O}$ NVQI-QOD Registry. ${ }^{1}$ Department of Neurological Surgery, Northwestern University Feinberg School of Medicine, Chicago, $L_{\text {; }}{ }^{2}$ Department of Radiology, Northwestern University Feinberg School of Medicine, Chicago, IL; ${ }^{3}$ Department of Radiology, Department of Neurological Surgery, Northwestern University Feinberg School of Medicine, Chicago, IL; ${ }^{4}$ Department of Radiology, Department of Neurological Surgery, Department of Neurology, Northwestern University Feinberg School of Medicine, Chicago, IL; ${ }^{5}$ SNIS, M2S, NPA, AANS, Fairfax, VA

\subsection{6/neurintsurg-2020-SNIS.276}

Introduction Sex differences in outcomes after acute ischemic stroke have been documented in the literature. However, few 